\title{
El papel de los modelos en la práctica científica'
}

\author{
The role of the models in the scientific practice \\ O papel dos modelos na prática científica
}

Recibido: diciembre de 2011 Aceptado: mayo de 2012
Rosa Nidia Tuay Sigua ${ }^{2}$

\section{Resumen}

Dos décadas de discusión en torno a los modelos como base para la investigación y la construcción del conocimiento científico han permitido abrir una ventana en un espacio oscurecido por la prevalecía dada a las teorías en sus importantes aproximaciones en el siglo XX. Este cambio de enfoque se debió, principalmente, a la gran atención que le han dado los filósofos a cómo se practica la ciencia en las múltiples disciplinas en las que los científicos investigan. El propósito de este artículo es hacer una descripción de las diferentes alternativas y posturas sobre las funciones de los modelos como representación y usos más frecuentes, enraizados en la combinación del trabajo conceptual y material que los científicos hacen, lo que permitirá tomar posturas acerca de las condiciones que seanmás adecuadas para abordar las dinámicas de la ciencia y su enseñanza.

Palabras clave: modelos, representaciones científicas, práctica científica.

\begin{abstract}
Two decades of discussion about models as a basis for research and scientific knowledge's advances have allowed to open a new way in an space, which was in darkness due to prevail of theories in its important approximations during XX century. According to this view, there is a big motivation to find new ways about science, not only less dependent of Theoric Physics epistemology, but also more accurate to real practices in physics and also in other sciences. The purpose of this article is to describe different alternatives and positions about model's functions as representation and its frequently uses, which are established between scientific conceptual and material's work. This view allows to take a position by the optimal conditions to tackle science's dynamics and its teaching.
\end{abstract}

Keywords: models, scientific representations, scientific practice.

\section{Resumo}

Duas décadas de discussão sobre modelos como base para a pesquisa e construção do conhecimento científico permitiram abrir uma janela num espaço obscurecido pela prevalência dada às teorias nas suas importantes aproximações no século XX. Esta mudança de enfoque se deveu, principalmente, a grande atenção que lhe deram os filósofos a como se pratica a ciência nas suas múltiplas disciplinas que os cientístas investigan.O

1 Artículo de investigación. Basado en la investigación realizada sobre modelos científicos en el Departamento de Física de la Universidad Pedagógica Nacional.

2 Universidad Pedagógica Nacional. Bogotá, Colombia. Contacto: rtuay@pedagogica.edu.co 
objetivo deste artigo é fazer uma descrição das diferentes alternativas e posturas sobre as funções dos modelos como representação e seus usos mais frequentes, enraizados na combinação do trabalho conceitual e material que os cientistas fazem, o que permitirá identificar posturas sobre as condições mais adequadas para abordar as dinâmicas da ciência e o seu ensino.

Palavras-chave: modelos, representações científicas, a prática científica.

\section{Introducción}

Cuando se asume que los modelos deben ser entendidos en su contexto de aplicación, estos surgen como una forma de la actividad de representación. Por esto, una gran cantidad de investigaciones se han dedicado a estudiar casos particulares de los modelos y de la modelización con gran detalle, orientados desde una relación diádica entre la fuente y el objetivo - target-. Éstas están expuestas en forma de similitud, causalidad, denotación y dejan de lado la relación triádica, donde es necesario introducir la intención del agente para dar cuenta del carácter representacional.

Las denominaciones de los modelos como aislamiento y razonamiento surrogativo, planteado desde la teoría económica, pretenden abordar las relaciones del modelo con el mundo a través de la epistemología. Se parte de la relación epistemológica de los modelos como análoga a la experimentación, donde: primero, se construye o se establece algo, después se investiga las propiedades de lo que se ha construido y luego se reflexiona sobre cómo se descubrieron las propiedades de lo construido en relación con el mundo. El razonamiento a través de modelos es esencialmente aprender sobre los sistemas de alquiler y esta naturaleza surrogativa diferencia la elaboración de modelos de otras actividades epistémicas como la representación directa. Es entonces lógico pensar que la epistemología de la modelización debe reflejar una característica esencial: aprender algo sobre los sistemas de construcción requiere una teoría adicional de cómo podemos asimilar algo acerca de la realidad mediante el aprendizaje alrededor de la construcción.

\section{Funciones delos modelos como representación}

Considerando la relación que se da entre la realidad y las teorías, se asume que los modelos pueden realizar dos funciones diferentes de representación:por una parte, un modelo puede ser una representación de una parte seleccionada del mundo;así, dependiendo de la naturaleza de la fuente, puede ser de fenómenos o de datos. De otra parte, un modelo puede representar una teoría en el sentido de interpretar los axiomas y las leyes de la teoría. Estas funciones no son excluyentes y los modelos pueden asumir cualquiera de las dos o las dos al mismo tiempo.

\section{Modelos de fenómenos}

Muchos modelos científicos representan un fenómeno, como el modelo de bola de billar de un gas, el del átomo de Böhr y el de doble hélice del ADN. El término "fenómeno" se utiliza para abordar los efectos repetibles y estables, o los procesos que son objetos potenciales de predicción y explicación sistemática de las teorías en general, y que pueden servir como prueba de estas. Realistas, como Bogen y Woodward (1988), no imponen ningún tipo de restricciones al presentar un "tercer nivel" en la dicotomía clásica de la teoría y las observaciones: los fenómenos científicos. De esto se diferencia la noción de fenómenos en la tradición empirista de "salvar los fenómenos, donde los observables son independientes de la teoría y depende de los individuos asumirlos como tal". Esta concepción es defendida por Van Fraassen (1980): "la creencia involucrada al aceptar una teoría científica está orientada a 'salvar los fenómenos', es decir, 'describir' correctamente lo que es observable".

Bogen y Woodward (1988) consideran que los fenómenos suelen ser observables. Sin embargo, los fenómenos se pueden deducir de los datos observables, después de que la fiabilidad de estos últimos han sido garantizados por los diversos procedimientos 
experimentales de la inferencia estadística, la reducción de datos, la exclusión de los factores de confusión, error y control de ruidos, y similares.

Hay otra característica que distingue a los datos y los fenómenos. Considerando que los fenómenos son "estables" en diferentes contextos experimentales, los datos suelen carecer de esta estabilidad y son a menudo muy particulares, debido a las causas propias de los contextos. Considerando que existen buenas razones para creer que hay un verdadero punto de fusión de plomo y que tiene un valor particular - es decir, $327,43{ }^{\circ} \mathrm{C}$-, puede darse que ninguno de los valores de un conjunto particular de lecturas del termómetro coincida con el valor real. Lo que es más, ni siquiera se puede saber la razón precisa de la variación de los datos. Estos dependen de una multitud de factores y no todos son susceptibles de controlar de forma plena.

Un modelo se considera como una descripción interpretativa de un fenómeno que facilita el acceso a éste. El fenómeno abarca tanto objetos como procesos. Muchas interpretaciones se basan, por ejemplo, en idealizaciones, simplificaciones o analogías con relación a las descripciones e interpretaciones de otros fenómenos. El acceso puede ser a través de la percepción o mediante la utilización de herramientas intelectuales. Facilitar el paso implica, generalmente, centrarse en aspectos concretos de un fenómeno, dejando deliberadamente de lado a otros. Como resultado de esto, los modelos sólo hacen descripciones parciales. Estos pueden ser objetos, como un avión de juguete y entidades abstractas o teóricas, por ejemplo el modelo estándar de la estructura de la materia y sus partículas fundamentales. En el primer caso, los modelos a escala permiten ampliar la percepción mediante la ampliación - comoun modelo de plástico de un copo de nieve- o la reducción - un globo como un modelo de la tierra-. Esto implica hacer explícitas las características que no son directamente observables - como la estructura de ADN o elementos químicos que hacen parte de la estructura de una estrella-.

La mayoría de los modelos científicos están lejos de ser algo material, como las barras y esferas de los modelos moleculares usados en la enseñanza.
Estos están relacionados con aspectos teóricos, pues con frecuencia se basan en ideas o conceptos abstractos y emplean un formalismo matemático - como los modelos del Bing Bang-, pero siempre tienen la intención de proporcionar acceso a los aspectos de un fenómeno que se consideran esenciales. Por ejemplo, el modelo del átomo de Böhr nos da una idea acerca de las configuraciones de los electrones y el núcleo de un átomo, así como de las fuerzas que actúan entre ellos -el modelo del corazón representado mediante una bomba nos da indicios acerca del funcionamiento de éste-.

Los medios a través de los cuales los modelos científicos se expresan van desde lo concreto a lo abstracto, como dibujos, diagramas, texto normales, gráficos, hasta ecuaciones matemáticas, por nombrar sólo algunos. Todas estas formas de expresión tiene el propósito de facilitar el acceso intelectual a las ideas relevantes que el modelo describe. Esto último, significa dar información y proveer recursos para interpretarlo, así como poder comunicar de manera eficiente a aquellos que comparten determinadas actividades intelectuales. Los modelos científicos se refieren particularmente a los fenómenos empíricos —objetos y procesos-, ya sea que se trate de cómo doblar y romper los metales,ode cómo fueron los procesos evolutivos del hombre.

Desde esta perspectiva, todo lo que se utiliza en ciencia para describir un fenómeno empírico es un modelo. Como los modelos científicos se refieren a tal variedad de entidades, a veces se dificulta decir algo que pueda ser común a todos los tipos de modelos científicos. Sin embargo, Frigg y Hartmann (2006) elaboraron unas categorías para abordar los estilos de representación propuestos en la literatura sobre los modelos. Entre ellos, están los modelos a escala, los idealizados, los analógicos y fenomenológicos. Estas categorías no son mutuamente excluyentes. Por ejemplo, algunos modelos a escala podrían considerarse también como idealizados y no está claro dónde trazar la línea divisoria entre los idealizados y los analógicos.

\section{- Modelos a escala}

Black (1962) considera que algunos modelos, por su tamaño, corresponden a copias ampliadas o 
reducidas de objetos. Por lo general, un modelo a escala es más pequeño que el objeto real. La relación entre el tamaño de un modelo y el del objeto real se llama escala. Los ejemplos típicos son los coches de colección o los modelos de puentes. Una primera intuición de una maqueta está en relación a una réplica naturalista o una imagen especular de un objeto. Por esta razón, los modelos a escala también se conocen como "modelos verdaderos", como lo expresa Achinstein (1968). Sin embargo, no existe una escala perfectamente fiel al modelo, pues la fidelidad es siempre restringida a algunos aspectos. El modelo del vehículo de colección, por ejemplo, proporciona una imagen fiel de la forma del coche, pero no dice nada acerca del material del que éste está construido.

En algunos casos, los modelos a escala se emplean como prototipos para predecir la función de un objeto. De esta manera, se procede sobre la base de ensayo y error, lo que no deja de ser un modo de cometer errores pero sin grandes costos y con cierta seguridad. Además, existen objetos que han de funcionar bien desde la primera vez y sólo se pueden probar con algún modelo. Por ejemplo, los túneles de viento fueron construidos por primera vez en el año 1871 por unos ingenieros ingleses para experimentar con las formas de las alas. Esto implica que la formulación de modelos a escala es menos obvia que sus usos. Para el caso del modelo de avión, aunque hayan parecidos entre el diseño del ala y eldel modelo a escala, es evidente los problemas que se enfrentan a su tamaño. La similitud sería condición para determinar los aspectos semejantes y diferentes. En todo caso, si se tuvieran dos modelos a escala de un mismo objeto, serían dos modos de representación distintos, pues se aplica directamente a los dos objetos físicos.

Los modelos a escala parecen ser un caso especial de una categoría más amplia de representaciones a las que Peirce (1931) llamó icónica: elementos que representan algo porque se parecen mucho. La representación icónica o de modelos a escala, por ejemplo, tiene un carácter local, en el sentido de que sólo se aplica a una situación particular en un momento determinado o que requiere la mediación de un modelo matemático - abstracto- para relacionarse con otras formas del discurso y la representación científica, como las teorías.

Los mapas se constituyen en una clase de modelos a escala, pues permitenla representación gráfica de la superficie de la tierra o parte de la misma, dibujada a escala o plano, donde los detalles se pierden. No tiene sentido un mapa a mayor escala, porque se volvería grande y detallado como el propio terreno, perdiendo así su utilidad.

De esta manera, los modelos a escala representan a su sistema de destino por el desplazamiento de una imagen idealizada y, la abstracción física de algunas de las características de éste y de sus relaciones.

\section{- Modelos analógicos}

El modelo analógico no es una reproducción detallada de todas las cualidades del sistema real, sino que refleja solamente la estructura de relaciones y determinadas propiedades fundamentales. Se establece una analogía entre el sistema real y el modelo, estudiándose el primero y utilizando como herramienta auxiliar al segundo. Se dice que dos cosas son análogas si hay ciertas similitudes relevantes entre ellas. Un ejemplo de esto lo encontramos en los computadores electrónicos, que han servido como modelos materiales de las operaciones intelectuales del ser humano.

Hesse (1963) considera que hay analogías materiales y formales. En una analogía material hay formas de establecer las clases de relaciones de similitud entre dos objetos. Una primera consideración es aquella que se basa en propiedades compartidas. Por ejemplo, en química, el término periódico significa repetición a intervalos regulares y en estas clasificaciones los elementos se han agrupado basándose en las similitudes de sus propiedades y en el incremento de los pesos atómicos. En algunos casos no es una condición necesaria la similitud entre sus propiedades, como en el caso de la analogía entre una célula vegetal y una fábrica. Para tener una idea de cuál es el funcionamiento global de una célula, se compara con lo que sucede en una fábrica: todas las partes de una fábrica no funcionan independientemente. 
La analogía entre dos objetos también puede basarse en las similitudes relevantes entre sus propiedades. En este sentido, es posible establecer una analogía entre la luz y el sonido, pues las reflexiones son similares a los ecos, el brillo al ruido; el color al tono, los órganos de detección, la detectabilidad visual a la detectabilidad auditiva, y así sucesivamente.

Las analogías también pueden basarse en la similitud o semejanza de las relaciones entre las partes de dos sistemas, en lugar de sus propiedades monovalentes. Este es el sentido en el que algunos políticos afirman que la relación entre un padre y sus hijos es análoga a la relación entre el Estado y los ciudadanos.

En la analogía formal se hace una abstracción de las características concretas que los sistemas poseen y sólo se centran en sus consideraciones formales. Lo que el modelo análogo comparte luego con su objetivo no es una serie de características, sino el mismo patrón de relaciones abstractas - por ejemplo, la misma estructura, donde ésta es entendida en el sentido formal-. Dos modelos están relacionados por analogía formal si ambos son interpretaciones del mismo cálculo formal. Hay una analogía formal entre un péndulo oscilante y un circuito eléctrico oscilante, debido a que ambos son descritos por la misma ecuación diferencial.

En las analogías formales, que se expresan entre sistemas que han sido modelados matemáticamente, las propiedades son las cantidades físicas que se usan en los modelos, mientras que las relaciones son las ecuaciones que los gobiernan. En una analogía, las variables en los modelos no precisan ser de la misma naturaleza:una variable en un modelo puede ser un vector, mientras que su correspondiente análogo puede ser un escalar. Pero, si todas las variables son de la misma naturaleza en los modelos y estos son análogos, entonces las ecuaciones son las mismas y se trata de un isomorfismo, pues ambas son interpretaciones del mismo cálculo formal.

Hesse (1974) también hace una clasificación de los modelos analógicos de acuerdo al valor positivo, negativo o neutro que se alcance entre dos modelos: una analogía positiva se da entre un modelo de las moléculas de un gas como bolas de billar, pues comparten una propiedad en común que es la masa. También se pueden compartir relaciones como en el caso de un péndulo y un circuito eléctrico oscilante. Estos sistemas son análogos en virtud de que sus relaciones formales se describen mediante la ecuación de onda. La 'A' en la fórmula resume lo que es común entre las ondas en el agua, las sonoras y las de la luz nos permite aplicar la amplitud del término.

En la analogía negativa no se comparten propiedades o relaciones:las bolas de billar son de colores, mientras que las moléculas del gas no lo son. La analogía neutral comprende las propiedades de las cuales no se tiene certeza si pertenecen a las analogías positivas o negativas. Un acercamiento a este tipo de analogía está dadoen relación a las moléculas del gas y si éstas cumplen las leyes de Newton: ¿se pude afirmar qué están en equilibrio?

\section{- Modelos fenomenológicos}

Los modelos fenomenológicos han sido definidos en diferentes maneras aunque todos relacionados entre sí. Una definición tradicional los toma como modelos que sólo representan propiedades observables de sus objetivos y se abstiene de postular mecanismos ocultos y similares. McMullin (1968) los define como aquellos que son independientes de las teorías. Muchos modelos fenomenológicos, mientras no pueden derivarse de una teoría, incorporan principios y leyes asociadas con teorías. Como el modelo de goteo liquido del núcleo del átomoque retrata al núcleo como una gota liquida y lo describe como poseedor de diversas propiedades - tensión de superficie y carga, entre otras-, lo que origina diferentes teorías - la hidrodinámica y electrodinámica, respectivamente- Ciertos aspectos de estas teorías - aunque usualmente no la teoría completa - son usados luego para determinar tanto las propiedades estáticas como las dinámicas del núcleo.

Portides (2006) considera que los modelos fenomenológicos son una especie de modelos matemáticos construidos por los físicos para aplicar la teoría a los fenómenos. Por tratarse de una especie de modelo matemático, su función de representación, 
su relación con la teoría y los fenómenos, su función como fuente de conocimiento, sus métodos de construcción $y$, en general, su importancia en la física se han visto opacados por otra especie de modelos matemáticos. Estos modelos se basan en la idea de que las teorías impulsadas por los modelos son un camino construido en una teoría sistemática regulada para completar el cálculo teórico a nivel local con las hipótesis operativas que se pueden expresar en un aparato conceptual, que se supone es congruente con la teoría.

Toda esta revisión muestra que los modelos de fenómenos se han comprendido en formas diversas en la ciencia y en la ciencia aplicada, y cómo los filósofos de la ciencia los analizan para clasificarlos en diferentes categorías. Los tipos y funciones de los modelos en la ciencia son demasiado diversos para que haya una noción unitaria que abarque a todos, y mucho menos que puedan ser confinados en una teoría formal como una camisa de fuerza.

\section{Modelos de datos}

Otro tipo de modelos de representación son los llamados "modelos de datos" (Suppes, 1962). En su trabajo, Suppes describe una jerarquía de modelos de datos que se conectan con la teoría. Propone tres niveles de modelos: los de la teoría, los de experimentación, y los de datos. Los modelos de la teoría y de experimentación están asociados con las teorías, mientras que los de datos describen las medidas recogidas en un experimento particular. Estos son los registros públicos - las fotografías de la cámara de burbujas, las fotografías de las posiciones estelares- producidos por la medición y el experimento, que sirven como prueba de la existencia de fenómenos o de la posesión de determinadas características. Los datos no sólo reflejan el influjo causal de los fenómenos que evidencia, sino también la operación de las características locales y la idiosincrasia de los dispositivos de medición, y el papel de los científicos expertos.

Por ejemplo, los modelos de datos contienen las lecturas de temperatura y presión tomadas durante un recorrido especial de un experimento para hallar la relación entre la temperatura y la presión de un gas cuando el volumen se mantiene constante. En la formulación original de Suppes, no todos los conjuntos de datos recogidos en un experimento se asumen como modelos de datos. Para que un conjunto de datos se constituya en un modelo de datos, deben darse condiciones en la teoría del experimento que puedan ser considerados dentro de un margen de error aceptable. También hay pruebas estadísticas que pueden realizar los conjuntos de datos para determinar si se constituye en un modelo de datos.

Los datos en bruto no son, por lo general, una forma utilizada por los científicos. Por esta razón, estos deben someterse a algún grado de procesamiento antes de que puedan ser analizados. Con frecuencia, un primer paso en el procesamiento de datos es la eliminación del ruido en su conjunto. Por ejemplo, si se están observando las posiciones de los planetas a través del cielo nocturno, se encuentra que hay un cierto grado de error asociado con cada una de las mediciones debido a defectos en el telescopio, el error humano, las condiciones atmosféricas $u$ otros factores en el medio ambiente. Antes de que se trace la trayectoria de un planeta determinado, es necesario hacer dos cosas: 1) la eliminación de algunos puntos que fueron resultado de un error - como ver un planeta donde no había uno solo-, y 2) dibujar una curva suave a través de los puntos restantes, de modo que la ruta de acceso resultante del planeta no es ir de punto a punto.

En resumen, el procesamiento de los datos permite producir un suave camino continuo para un planeta a partir de una colección de datos discretos. Los recuentos de la información, como los datos en bruto, deben hacerse casi siempre por medio de este tipo de tratamiento antes de que tenga una forma utilizable. Este último trazado de la ruta de acceso del planeta puede ser considerado un modelo de datos. Muchos cambios se han hecho en el conjunto de datos original. Algunos puntos de datos han sido eliminados, y los valores discretos han sido sustituidos por una curva suave y continua. El primer paso se llama reducción de datos, y el segundo, ajuste de curvas. Estos dos pasos se utilizan a menudo cuando se construye tramas de datos en los laboratorios de química o 
física, como la temperatura en función de la resistividad en un experimento de física del estado sólido, o latemperatura en función de la presión en un test de la ley de los gases ideales. Las curvas resultantes no sólo son similares a los datos originales, sino también diferentes, siendo continuas $y$, por lo general, no coinciden exactamente con la mayoría de los puntos originales en los datos. Las curvas son ahora un modelo de datos construido por los científicos y son similares a los datos originales en los aspectos relevantes.

El empleo, por parte de las diferentes ciencias, de diversos tipos de modelos para representar fenómenos de muy distinta naturaleza se constituye en una práctica implementada por la comunidad científica para dar cuenta de un sinnúmero de eventos, regularidades y construcciones que les permite comprender el mundo con la ayuda de estos.

Los científicos han reconocido la importancia de la modelización en la ciencia contemporánea, de tal manera que los modelos pueden ser asumidos como mapas, mundos, experimentos, objetos, construcciones sociales, donde la mayoría de estas interpretaciones busca la captura de un aspecto importante de ellos sin estar necesariamente en mutua contradicción. Los modelos no pertenecen a una clase natural. La elección de aspectos como piedras angulares de un enfoque de los modelos científicos es, en cierta medida, subjetiva y sólo se justifica en términos de su contribución a la aclaración de aspectos relevantes.

Las investigaciones en torno a la enseñanza de las ciencias que han utilizado como base las construcciones de modelos, no han logrado llegar a consensos sobre el significado y el alcance del término "modelo". Sin embargo, se está de acuerdo en que el modelo es un "sustituto"o un "subrogado"de los sistemas reales que se estudian, demasiado complejos para poder ser abordados en su totalidad; los científicos trabajan con las "representaciones"de estos sistemas que conservan sólo sus aspectos esenciales. Es por eso que los modelos actúan como facilitadores para la comprensión del mundo real.

\section{Conclusiones}

El renovado interés por el estudio de los modelos se manifiesta en la enorme cantidad de publicaciones que van más allá del análisis y el establecimiento de taxonomías acerca de los variados usos, funciones, naturalezas, y se dirigen hacia consideraciones sobre su papel en la práctica científica.

Los modelos, de acuerdo a la revisión que se ha presentado, pueden ser interpretados como objetos diversos y variados - mapas, mundos, experimentos, objetos, construcciones sociales, y así sucesivamente-, y la mayoría de estas interpretaciones dan cuenta de sus principales características, sin que haya necesariamente una mutua contradicción. Es indiscutible que los modelos no constituyen algo así como un "reino natural". La elección de cada uno de los aspectos que caracterizan a los enfoques sobre los modelos científicos es, en cierto modo, subjetivo y sólo puede ser justificado en la medida que contribuyen a dilucidar el resto de los aspectos pertinentes.

La elaboración de modelos, relativamente independientes entre sí y que permitan expresar coherentemente los datos empíricos, ha tenido una creciente importancia filosófica, en la que se destacan:el problema de las relaciones entre modelos y la realidad, el problema de la evaluación, y la interpretación ontológica y epistemológica de los modelos científicos.

La mayoría de los trabajos se han centrado en la concepción semántica, demostrando que se pueden abordar aspectos interesantes con una economía de medios formales, pues se habla directamente de las estructuras y de los modelos de las teorías, en lugar de formulaciones lingüísticas. Sin embargo, trabajos recientes como los de Donato y Zamora (2009) han insistido en hallar puntos de contacto entre la tradición semántica y los estudios metodológicos y epistemológicos orientados desde aspectos pragmáticos de la investigación científica, donde el interés principal es comprender las decisiones de los científicos.

El empleo de diversos tipos de modelos para representar fenómenos de muy distinta naturaleza, por 
parte de las diferentes ciencias, se constituye en una práctica implementada por la comunidad científica y académica para dar cuenta de un sinnúmero de eventos, regularidades y construcciones para el mundo. La enseñanza por modelización pretende que los estudiantes tengan la capacidad de pensar teóricamente, de interpretar lo que se ve y se toca, de intervenir en ello y de preveer fenómenos futuros, en términos de entidades abstractas (Izquierdo, 2004).

El estudio de los modelos y la modelización busca comprender cuál es el proceso de construcción y de cambio de las representaciones y, así mismo, proponer, aplicar y evaluar nuevas propuestas curriculares en las que esta perspectiva de enseñanza y aprendizaje se aplique. Se requiere, entonces, conocer las representaciones de los estudiantes - llámense ideas previas, modelos mentales, u otras acepciones-, cómo están presentes en su mente y cómo son usadas por los sujetos para su razonamiento y para la resolución de problemas de su cotidianidad. El desconocimiento de estos aspectos dificultaría conseguir transformaciones de las representaciones por parte de los estudiantes en los procesos de enseñanza y aprendizaje (Tuay 2012).

\section{Referencias}

Achinstein, P. (1968). Concepts of Science: A Philosophical Analysis. Baltimore: Johns Hopkins Press.

Black, M. (1962). Models and Metaphors. Ithaca: Cornell University Press.

Bogen, J. and Woodward, J. (1988). "Saving the Phenomena”. Philosophical Review. (97): 303-352.

De Donato, X. and Zamora Bonilla, J. (2009). "Credibility, Idealisation, and Model Building: An Inferential Approach". Erkenntnis. (70): 101-118. Doi: 10.1007/s10670-008-9139-5

Frigg, R and Hartmann, S. (2006). Models in Science. The Stanford Encyclopedia of Philosophy. Available: http://plato.stanford. edu/entries/models-science/
Hesse, M. (1963). Models and analogies in science. London: Publisher.

Hesse, M. (1964). "Analogy and Confirmation Theory". Philosophy of Science. (31): 319-327.

Izquierdo, M. (2004). "Un nuevo enfoque de la enseñanza de la química: contextualizar y modelizar". The Journal Argentine Chemical Society. (92): 115-136.

McMullin, E. (1968). What Do Physical Models Tell Us? In: B. van Rootselaar and J. F. Staal. (Eds.). Logic, Methodology and Science III. Amsterdam: North Holland.

Peirce, C. (1931). Collected Papers.(Volume 2). In:Hartshorne, C. y Weiss, P. Elements of Logic. Cambridge: Harvard University Press.

Portides, D. (2005). "Scientific Models and the Semantic View of Scientific Theories". Philosophy of Science. (72): 1287-1298.

Suppes, P. (1962). Models of Data. In: E. Nagel; P. Suppes and A. Tarski.(Eds.). Logic, Methodology and Philosophy of Science. (Proceedings of the 1960 International Congress. Stanford: Stanford University Press. Reprinted in: Suppes, P. Studies in the Methodology and Foundations of Science. Selected Papers from 1951 to 1969. Dordrecht: Reidel.

Tuay, R. (2007). “Aproximación al Debate de las Representaciones Científicas". Revista Lindaraja. (11). Disponible en: http://www. filosofiayliteratura.org/Lindaraja/nidia/nidia_tuay.htm.

Tuay, R. (2012). "La construcción y uso de los modelos en las ciencias naturales y su didáctica". Revista Internacional Magisterio. (57): 60-65.

Van Fraassen, B. (1980). The Scientific Image. Oxford: Clarendon Press.

Van Fraassen, B. (1996). Science, Materialism, and False Consciousness. In:Contemporary Epistemology: Essays in Honor of Alvin Plantinga's Theory of Knowledge. Warrant: Rowman Littlefield. 
Van Fraassen, B. (2008). Scientific Representation: Paradoxes of Perspective. Oxford: Oxford University Press.

Zamora Bonilla, J. (2003). La lonja del saber. Introducción a la economía del conocimiento científico. Madrid: Servicio de publicaciones de la UNED.

Zamora Bonilla, J. (2005). Cuestión de protocolo. Ensayos de metodología de la ciencia. Madrid: Tecnos.
Zamora Bonilla, J. (2005). Ciencia Pública-Ciencia Privada. Reflexiones sobre la producción del saber científico. Madrid: Fondo de Cultura Económica.

Zamora Bonilla, J. (2006a). "Science as a persuasion game”. Episteme. (2): pp. 189-201.

Zamora Bonilla, J. (2006b). "Science studies and the theory of games". Perspectives on Science. (14): pp. 639-671. 